\title{
Checklist do filo Chaetognatha do Estado de São Paulo, Brasil
}

\author{
Luz Amelia Vega-Pérez ${ }^{1,2}$ \& Katya Patrícia Schinke ${ }^{1}$ \\ ${ }^{1}$ Departamento de Oceanografia Biológica, Instituto Oceanográfico, Universidade de São Paulo - USP, \\ Praça do Oceanográfico, n. 191, Butantã, CEP 05508-120, São Paulo, SP, Brasil \\ ${ }^{2}$ Autor para correspondência: Luz.Amelia Vega-Pérez, e-mail: lavega@usp.br
}

VEGA-PÉREZ, L.A. \& SCHINKE, K.P. Checklist of chaetognatha phyllum from São Paulo State, Brazil. Biota Neotrop. 11(1a): http://www.biotaneotropica.org.br/v11n1a/en/abstract?inventory+bn0241101a2011.

\begin{abstract}
The species of Chaetognatha, commonly called arrow-worms, are considered one of the most taxonomically isolated animal groups, with obscure phyletic origin. They are deuterostomes of small size, between 2 and $12 \mathrm{~mm}$, with bilateral symmetry and transparent torped-shaped body, although some species have pigmentation. The body consists of the head, the trunk and the tail. The head bears a ventrally placed mouth, surrounded by two sets of rigid hooks and rows of small teeth, both used in prey capture. There are two dorsal eyes, which are absents in some deep living species. The trunk bears paired lateral fins and the tail a single fin. They are protandrous hermaphrodites with direct development, being the cross-fertilization probably typical in this phylum. Fertilization is internal and the eggs released directly into the water. Although chaetognaths are eaten by numerous larger carnivorous organisms, in the food web they are important predators and a significant trophic link between small herbivores and larger predators, including important commercial fish species. Cannibalism is known, particularly in some species. They have been recognized as important producers of large quantities of fecal pellets, which must play a significant role in the flux of organic carbon in the oceans. Chaetognaths are also considered good indicators of potentially important fishery areas, and more recently, they gained recognition as vectors in the life cycles of various parasite groups. They are exclusively marine and can be found in all oceans from surface to great depths and in estuarine regions. Generally are most abundant around 100-200 m depths. With exception to the epibenthic family Spadellidae, the chaetognaths are conspicuous members of the zooplankton. Their distribution pattern is influenced by the hydrobiological conditions and some species are known as indicators of water masses. A total of 209 species were recorded in the world's oceans, and 29 for the South Atlantic. Twenty five species are known from the Brazilian waters and only 14 species from coastal and offshore waters of São Paulo State.
\end{abstract}

Keywords: chaetognatha, biodiversity of the State of São Paulo, BIOTA/FAPESP Program.

Number of species: in the world: 209, in Brazil: 25, estimated in São Paulo State: 25.

VEGA-PÉREZ, L.A. \& SCHINKE, K.P. Checklist do filo chaetognatha do Estado de São Paulo, Brasil. Biota Neotrop. 11(1a): http://www.biotaneotropica.org.br/v11n1a/pt/abstract?inventory+bn0241101a2011.

Resumo: O filo Chaetognatha é constituído de um pequeno número de animais marinhos deuterostomados, filogeneticamente isolados, de simetria bilateral e corpo alongado em forma de torpedo ou seta, cujo comprimento pode variar de 2 a $120 \mathrm{~mm}$. O corpo é dividido em três regiões: cabeça, tronco e cauda. A cabeça apresenta um par de olhos e a boca situada no vestíbulo ventral, a qual é circundada por uma coroa de espinhos fortes e por uma ou duas fileiras de dentes. O tronco possui um ou dois pares de nadadeiras laterais e a cauda uma nadadeira na extremidade posterior. São hermafroditas protândricos sendo em geral a fecundação cruzada, interna, e o desenvolvimento direto. São predadores que se alimentam de uma grande variedade de organismos. O canibalismo também é observado no grupo. Os quetógnatos desempenham papel fundamental na teia trófica marinha, como carnívoros primários e item alimentar de organismos planctófagos especialmente peixes de interesse comercial. São considerados bons indicadores de áreas de pesca, potencialmente importantes, grandes produtores de matéria orgânica particulada e peça chave no fluxo do carbono nos oceanos. Mais recentemente, foram reconhecidos como hospedeiros intermediários no ciclo de vida de vários grupos de parasitas marinhos. Os quetógnatos são encontrados em todos os mares, oceanos e regiões estuarinas, sendo mais abundantes entre os 100 e $200 \mathrm{~m}$ de profundidade. Com exceção dos componentes da família Spadellidae, que agrupa espécies bentônicas, a grande maioria é planctônica. A distribuição desses organismos é influenciada em geral pelas condições hidrológicas, sendo algumas espécies usadas como indicadoras de massas de água. No momento, 209 espécies de Chaetognatha foram descritas no mundo, das quais 29 ocorrem no Atlântico Sul e 25 em águas brasileiras. No estado de São Paulo, apenas, 14 espécies foram encontradas nas regiões costeiras e oceânicas.

Palavras-chave: chaetognatha, biota paulista, Programa BIOTA/FAPESP.

Número de espécies: no mundo: 209, no Brasil: 25, estimadas no Estado de São Paulo: 25. 


\section{Introdução}

O filo Chaetognatha é constituído de um pequeno número de animais marinhos deuterostomados, filogeneticamente isolados, de simetria bilateral marcada e corpo alongado em forma de torpedo ou seta. O nome deriva do grego "Ghaite" que significa espinho e "Gnathos" mandíbula, maxila.

O corpo, cujo comprimento pode variar de 2 a $120 \mathrm{~mm}$, é dividido por septos transversais em três regiões: cabeça, tronco e cauda. A cabeça, que tem forma triangular e é separada do tronco por um estreito pescoço, apresenta um par de olhos pigmentados invertidos na parte posterior da superfície dorsal, e a boca situada no vestíbulo ventral. A boca é circundada por uma coroa de espinhos curvados fortes e por uma ou duas fileiras de pequenos dentes quitinosos. $\mathrm{O}$ número e formato dessas duas estruturas variam de espécie para espécie, podendo diminuir ou serem perdidos em animais mais velhos. A dobra antero-lateral da parede do corpo forma um capuz retrátil que serve para envolver e proteger o vestíbulo.

O tronco possui um ou dois pares de nadadeiras laterais, cuja parte posterior prolonga-se até o segmento caudal. Este último apresenta o celoma dividido por um septo longitudinal e uma nadadeira de forma espatulada na extremidade posterior. As nadadeiras são enrijecidas por duas fileiras de "raios" quitinosos, derivados aparentemente da membrana basal da epiderme.

As espécies pelágicas são transparentes, translúcidas, enquanto as que habitam as camadas mais profundas podem apresentar coloração rosa, azul clara, amarelada, avermelhada e em alguns casos cor marrom. A bioluminescência é um fenômeno raro no grupo, restringindo-se a uma ou duas espécies.

O trato digestivo dos quetógnatos é simples, formado pela boca, a faringe muscular e bulbosa, o intestino reto e o ânus ventral localizado na junção tronco-cauda. Não possuem órgãos excretores ou para troca gasosa, sendo esta realizada por meio de difusão. Os gânglios cerebral (cabeça) e ventral (subentérico), que são grandes e se interligam por conectivos circum-entéricos, atuam como centros nervosos, enquanto o fluido celômico como meio circulatório. Os quetógnatos apresentam no corpo cerdas ciliares que servem para detectar as vibrações geradas na água, e uma alça ciliada ou "corona ciliata" anterior de função incerta. Esses organismos caracterizam-se também por apresentar músculos longitudinais atrofiados, arranjados em quadrantes, e pela ausência de musculatura circular (Hyman 1959, Boltovskoy 1981a, Bone et al. 1991, Casanova 1999, Brusca \& Brusca 2007).

São hermafroditas protândricos, sendo o aparelho reprodutor constituído por um par de ovários alongados localizados no celoma do tronco e por um par de testículos na região caudal. De um modo geral, a fecundação é cruzada, interna, sendo a autofecundação praticada por alguns grupos. Os ovos fertilizados são liberados no ambiente onde podem flutuar, quando rodeados de uma substância gelatinosa, aderir-se a objetos imóveis ou afundar. Algumas espécies de Eukrhonia que habitam águas profundas carregam os embriões em duas bolsas de natureza gelatinosa ("marsúpio"), localizadas próximo à cauda, até a eclosão. $\mathrm{O}$ desenvolvimento é direto e tem aproximadamente 48 horas de duração. Independente da época do ano e do local, as populações de quetógnatos são constituídas basicamente de estágios jovens, o que indica que a reprodução ocorre o ano todo (Alvariño 1963, Fagetti 1968, Owre 1960, Reeve 1970, Duró \& Gili 2000, Liang \& Vega-Pérez 2001, 2002, Liang et al. 2003).

Os quetógnatos são carnívoros vorazes que se alimentam de uma grande variedade de organismos incluindo ovos e larvas de peixes, sendo os copépodes o item principal na sua dieta. O canibalismo intra e interespecífico também é observado no grupo. O tipo de alimento consumido reflete de um modo geral a composição do zooplâncton local e está relacionado à abundância, tamanho, padrão de migração da presa, idade e tamanho do predador (Reeve \& Walter 1972, Pearre 1976, Terazaki \& Marumo 1982, Alvariño 1985, Øresland 1990, Liang 1998, Liang \& Vega-Pérez 1995, 2001).

Algumas espécies são parasitadas por Annelida, Nematoda e Trematoda que se localizam geralmente no trato digestivo ou no celoma do corpo (Pierrot-Bults 1996, Del Prado-Rosas et al. 2005, Øresland \& Bray 2005, Daponte et al. 2008), outros carregam epiparasitas especialmente no tronco (Vega-Pérez \& Schinke 2008).

Os quetógnatos possuem ampla distribuição, sendo encontrados em todos os mares e oceanos do mundo, bem como em regiões estuarinas e mangues. Com exceção dos componentes da família Spadellidae, que agrupa espécies bentônicas, a grande maioria é planctônica. São frequentes e abundantes em regiões neríticas e oceânicas, entre os 100 e $200 \mathrm{~m}$ de profundidade, sendo a fauna mais diversificada nas camadas de 0 a 300 m e 800 a 1000 m (Pierrot-Bults \& Nair 1991, Hossfeld 1996, Casanova 1999).

Em geral, a distribuição desses organismos é limitada pelas propriedades das massas de água devido à sua sensibilidade às variações de temperatura e salinidade (Alvariño 1969, Cheney 1985a,b, McLelland 1984, 1991). Assim, algumas espécies são consideradas boas indicadoras de massas de água, contribuindo juntamente com outros organismos para delimitar regiões biogeográficas marinhas (Russel 1939, Bieri 1959, Alvariño 1965, 1969, Boltovskoy 1981b, Bieri 1991).

Os quetógnatos são um dos principais componentes do ecossistema marinho devido ao papel que desempenham na teia trófica, como carnívoros primários e item alimentar de organismos planctófagos especialmente peixes de interesse comercial. Em certos locais e épocas do ano, devido à sua abundância e hábitos alimentares podem regular o tamanho da população-presa e competir com as larvas de peixes pelo alimento, afetando com isso o recrutamento desses organismos. Além disso, o fato de serem grandes produtores de matéria orgânica particulada os torna peça chave no fluxo do Carbono nos oceanos (Pearre 1980, Nagasawa \& Marumo 1981, Feigenbaum \& Maris 1984, Øresland 1990, Dilling \& Alldredge 1993, Terazaki 1998, Bone et al. 1991).

De acordo com o histórico detalhado feito por Kuhl (1938) e Hyman (1959), o primeiro desenho de um Chaetognatha foi feito por Slabber em 1774, quem os chamou de verme seta ou sagitta. Quoy \& Gaimard (1927) fizeram uma breve descrição de espécimes coletados no Estreito de Gibraltar em 1826, porém, sem fornecer nenhuma figura desses organismos. No entanto, nenhum desses autores soube como classificar esses organismos. No século XIX, numerosos artigos foram publicados abordando aspectos relacionados com a anatomia, embriologia, histologia e sistemática dos quetógnatos. Foi Leuckart (1854 In: Hyman, 1959) quem os denominou Chaetognathi e sugeriu que fossem colocados em um grupo separado, entre Annelida e Nematoda. Os estudos de embriologia feitos por Genenbaur (1858 In: Hyman, 1959) permitiram que em 1870 o autor aceitasse o nome proposto por Leuckart. Posteriormente, esse nome foi modificado para Chaetognatha (Hyman 1959).

O problema das afinidades do filo Chaetognatha foi discutido por diversos autores como Kuhl (1938), Hyman (1959), Ghirardelli (1968), Ducret (1968) e Casanova (1985, 1986, 1987). Contudo, a origem e o desenvolvimento evolutivo do grupo ainda são desconhecidos (Kapp, 2005).

A primeira coleta de espécimes desse filo no Atlântico Sul foi realizada por Darwin em 1833 durante a expedição a bordo do "Beagle". Com base nos dados obtidos, Darwin (1844) concluiu que os quetógnatos eram abundantes nas regiões afastadas das costas do Brasil, Argentina e Chile. Os trabalhos pioneiros abordando aspectos da sistemática e distribuição das espécies que ocorrem em águas brasileiras foram feitos a partir de amostras de plâncton obtidas 
durante as expedições oceanográficas, esporádicas e espaçadas, realizadas por navios estrangeiros. Dentre esses destacam-se: "Nave Liguria" (Baldasseroni, 1915), "Meteor" (Thiel 1938) e "Terra Nova" (Burfield 1930). Posteriormente, outros trabalhos foram realizados por Vanucci \& Hosoe (1952), Hosoe (1956), Alvariño (1980), Gusmão (1986), Nair \& Sankarankutti (1988), Liang (1998), Liang \& Vega-Pérez (2001), Sousa \& Lopes (1998), Araújo \& Ribeiro (2005), Loureiro et al. (2005) e Ávila et al. (2006), que estudaram os quetógnatos coletados na costa leste e central do Brasil, enquanto Almeida-Prado (1960, 1961a,b, 1963, 1968), Costa (1970, 1971), Coelho (1993), Liang (1993), Liang \& Vega-Pérez (2002), Resgalla \& Montú (1995), Marazzo-Nogueira et al. (1996), Vega-Pérez \& Liang (1999), Liang et al. (2003) e Vega-Pérez \& Schinke (2008), estudaram a ocorrência e distribuição das que ocorrem nas regiões sudeste e sul. Aspectos relacionados aos hábitos alimentares dos quetógnatos foram abordados por Almeida-Prado (1968), Vega-Pérez \& Liang (1992, 2004), Liang \& Vega-Pérez (1995) e Marazzo et al. (1997).

O filo no momento é formado por 209 espécies, sendo a família Sagittidae a mais bem sucedidas pois reúne o maior número de gêneros e espécies (Alvariño 1969, Bieri 1991, Bone et al. 1991, Casanova 1999). No Brasil o filo é representado por 25 espécies (Tabela 1), das quais 14 ocorrem no Estado de São Paulo, o que evidencia que a fauna de Chaetognatha no país ainda é pouco conhecida.

\section{Metodologia}

A lista das espécies de Chaetognatha que ocorrem no Estado de São Paulo (Tabelas 1 e 2) foi elaborada baseada nos trabalhos de: Almeida Prado (1960; 1961a,b, 1968), Alvariño (1969, 1980), Boltovskoy (1981a), Liang (1993, 1998), Liang \& Vega-Pérez
(1994, 2001, 2002), Vega-Pérez \& Schinke (2008). Além dessas referências foram consultados os seguintes sites: World Register of Marine Species - WORMS (2010): www.marinespecies.org/. e Ocean Biogeographic Information System - OBIS (2010): www.iobis.org/.

\section{Resultados e Discussão}

\section{Espécies de Chaetognatha que ocorrem nas águas do Estado de São Paulo}

\author{
Filo Chaetognatha \\ Ordem Aphragmophora \\ Família Sagittidae Claus and Grobben, 1905 \\ Ferosagitta hispida (Conant, 1895) \\ Flaccisagitta enflata (Grassi, 1881) \\ Flaccisagitta hexaptera (d'Orbigny, 1836) \\ Mesosagitta minima (Grassi, 1881) \\ Parasagitta friderici Ritter-Záhony, 1911 \\ Parasagitta tenuis (Conant, 1896) \\ Pseudosagitta lyra (Krohn, 1853) \\ Pseudosagitta maxima (Conant, 1896) \\ Sagitta bipunctata Quoy \& Gaimard, 1828 \\ Serratosagitta pacifica (Tokioka, 1940) \\ Serratosagitta serratodentata (Krohn, 1853) \\ Família Pterosagittidae Tokioka, 1965 \\ Pterosagitta draco (Krohn, 1853) \\ Família Krohnittidae Tokioka, 1965 \\ Krohnitta pacifica (Aida, 1897) \\ Krohnitta subtilis (Grassi, 1881)
}

Tabela 1. Espécies de Chaetognatha que ocorrem nas águas brasileiras.

Table 1. Species of Chaetognatha that occur in Brazilian waters.

\begin{tabular}{|c|c|c|c|c|c|c|c|c|}
\hline Classificação & $\begin{array}{c}\text { Taxon } \\
\text { superior }\end{array}$ & Gênero & $\begin{array}{c}\text { Epíteto } \\
\text { específico }\end{array}$ & Descritor & $\begin{array}{c}\text { Ano da } \\
\text { descrição }\end{array}$ & $\begin{array}{l}\text { Coleção } \\
\text { científica }\end{array}$ & $\begin{array}{c}\mathrm{N}^{\circ}{ }^{\circ} \text { do } \\
\text { registro }\end{array}$ & Coletor \\
\hline \multirow[t]{25}{*}{ F. Chaetognatha } & O. Aphragmophora & Caecosagitta & macrocephala & (Fowler) & $(1905)$ & - & - & Ávila et al. 2004 \\
\hline & & Decipisagitta & decipiens & (Fowler) & (1905) & - & - & Coelho 1993 \\
\hline & & Decipisagitta & sibogae & (Fowler) & (1906) & - & - & Ávila et al 2004 \\
\hline & & Ferosagitta & hispida $*$ & (Conant) & $(1895)$ & - & - & Vega-Pérez 1986-2009 \\
\hline & & Flaccisagitta & enflata * & (Grassi) & $(1881)$ & - & - & Vega-Pérez 1987-2009 \\
\hline & & Flaccisagitta & hexaptera* & (d’Orbigny) & $(1836)$ & - & - & Vega-Pérez 1987-2009 \\
\hline & & Mesosagitta & $\operatorname{minima} *$ & (Grassi) & $(1881)$ & - & - & Vega-Pérez 1987-2009 \\
\hline & & Parasagitta & friderici $*$ & Ritter-Záhony & 1911 & - & - & Vega-Pérez 1987-2009 \\
\hline & & Parasagitta & tenuis * & (Conant) & $(1896)$ & - & - & Vega-Pérez, 1987-2009 \\
\hline & & Pseudosagitta & gasellae & (Ritter-Zahóny) & (1909) & - & - & Alvariño 1980 \\
\hline & & Pseudosagitta & lyra* & (Krohn) & $(1853)$ & - & - & Vega-Pérez 1987-2009 \\
\hline & & Pseudosagitta & maxima $*$ & (Conant) & $(1896)$ & - & - & Vega-Pérez 1987-2009 \\
\hline & & Sagitta & bipunctata* & Quoy \& Gaimard & 1828 & - & - & Vega-Pérez 1987-2009 \\
\hline & & Sagitta & helenae & Ritter-Záhony & 1911 & - & - & Almeida-Prado 1960 \\
\hline & & Serratosagitta & pacifica $*$ & (Tokioka) & $(1940)$ & - & - & Vega-Pérez 1987-2009 \\
\hline & & Serratosagitta & serratodentata* & (Krohn) & $(1853)$ & - & - & Vega-Pérez 1987-2009 \\
\hline & & Serratosagitta & tasmanica & (Thompson) & (1947) & - & - & Coelho 1993 \\
\hline & & Solidosagitta & planctonis & (Steinhaus) & $(1896)$ & - & - & Coelho 1993 \\
\hline & & Solidosagitta & zetesios & (Fowler) & $(1905)$ & - & - & Alvariño 1980 \\
\hline & & Pterosagitta & draco * & (Krohn) & $(1853)$ & - & - & Vega-Pérez 1987-2009 \\
\hline & & Krohnitta & pacifica $*$ & (Aida) & $(1897)$ & - & - & Vega-Pérez 1987-2009 \\
\hline & & Krohnitta & subtilis $*$ & (Grassi) & $(1881)$ & - & - & Vega-Pérez 1987-2009 \\
\hline & & Eukrohnia & bathypelagica & Alvariño & 1962 & - & - & Resgala \& Montú 1995 \\
\hline & & Eukrohnia & hamata & (Mörbius) & $(1875)$ & - & - & Coelho 1993 \\
\hline & O. Phragmophora & Paraspadella & sp. & Salvini-Plawen & 1986 & - & - & Ávila et al. 2004 \\
\hline
\end{tabular}

(*) ocorrem no Estado de São Paulo. 
Tabela 2. Checklist das espécies de Chaetognatha que ocorrem ao largo do Estado de São Paulo.

Table 2. Checklist of Chaetognatha species found on the coast of São Paulo state.

\begin{tabular}{|c|c|c|c|c|c|c|c|c|}
\hline Classificação & Taxon Superior & Gênero & $\begin{array}{c}\text { Epíteto } \\
\text { específico }\end{array}$ & Descritor & $\begin{array}{c}\text { Ano da } \\
\text { descrição }\end{array}$ & $\begin{array}{c}\text { Coleção } \\
\text { científica }\end{array}$ & $\begin{array}{c}\mathrm{N}^{\circ}{ }^{\circ} \text { do } \\
\text { registro }\end{array}$ & Coletor \\
\hline \multirow[t]{14}{*}{ F. Chaetognatha } & O. Aphragmophora & Ferosagitta & hispida & (Conant) & $(1895)$ & - & - & Vega-Pérez 1987 \\
\hline & & Flaccisagitta & enflata & (Grassi) & (1881) & - & - & Vega-Pérez 1987 \\
\hline & & Flaccisagitta & hexaptera & (d’Orbigny) & (1836) & - & - & Vega-Pérez 1987 \\
\hline & & Mesosagitta & minima & (Grassi) & (1881) & - & - & Vega-Pérez 1987 \\
\hline & & Parasagitta & friderici & Ritter-Záhony & 1911 & - & - & Vega-Pérez 1987 \\
\hline & & Parasagitta & tenuis & (Conant) & (1896) & - & - & Vega-Pérez 1987 \\
\hline & & Pseudosagitta & lyra & (Krohn) & (1853) & - & - & Vega-Pérez, 1997 \\
\hline & & Pseudosagitta & maxima & (Conant) & (1896) & - & - & Vega-Pérez 1987 \\
\hline & & Sagitta & bipunctata & Quoy \& Gaimard & 1828 & - & - & Vega-Pérez 1987 \\
\hline & & Serratosagitta & pacifica & (Tokioka) & (1940) & - & - & Vega-Pérez 1987 \\
\hline & & Serratosagitta & serratodentata & (Krohn) & (1853) & - & - & Vega-Pérez 1987 \\
\hline & & Pterosagitta & draco & (Krohn) & (1853) & - & - & Vega-Pérez 1987 \\
\hline & & Krohnitta & pacifica & (Aida) & (1897) & - & - & Vega-Pérez 1987 \\
\hline & & Krohnitta & subtilis & (Grassi) & (1881) & - & - & Vega-Pérez 1987 \\
\hline
\end{tabular}

\section{Comentários sobre a lista, riqueza do Estado comparado com outras regiões}

A fauna de Chaetognatha do estado de São Paulo apresenta de um modo geral composição específica semelhante à relatada para outras regiões do mundo e, especialmente, às do Sul-Sudeste e Nordeste do Brasil. Os trabalhos publicados evidenciam que São Paulo é um dos estados que possuem a fauna melhor conhecida e representada, uma vez que detém 14 (56\%) das 25 espécies assinaladas para as águas brasileiras. Se levarmos em consideração o total desconhecimento da fauna bentônica de São Paulo, é muito provável que esse número seja facilmente superado. Isso, porque em outras regiões do mundo já foram descritas pouco mais de 30 espécies de águas profundas e bentônicas (Casanova 1999, Kasatkina \& Selivanova 2003, Cassanova et al. 2006, Tovar \& Suárez-Morales 2007).

\section{Principais avanços relacionados ao Programa BIOTA/FAPESP}

Apesar dos esforços realizados pelas instituições envolvidas no Programa BIOTA/FAPESP, no tocante ao conhecimento dos invertebrados marinhos do Estado de São Paulo, podemos afirmar que poucos são os avanços obtidos para o filo Chaetognatha nestes últimos dez anos. Esse fato pode ser comprovado pelo reduzido número de trabalhos publicados e, principalmente, pela ausência de monografias, dissertações e teses defendidas nesse período em São Paulo.

\section{Principais grupos de pesquisa}

O número de pesquisadores dedicados ao estudo da fauna de Chaetognatha no Brasil é bastante reduzido. No momento, no Estado de São Paulo apenas dois pesquisadores pertencentes ao Instituto Oceanográfico da Universidade de São Paulo vêm estudando as espécies que ocorrem no Sistema Estuarino da Baixada Santista, da região de Ubatuba e São Sebastião.

\section{Principais acervos}

No país, no momento, não se conta com coleções de referência completas de Chaetognatha. As coleções existentes pertencem aos próprios pesquisadores, ou estão sob sua guarda, e são mantidas muitas vezes no seu ambiente de trabalho. Várias instituições de ensino, ainda, contam com coleções didáticas.
Algumas espécies de Chaetognatha planctônicos retirados de amostras de zooplâncton, pertencentes ao acervo do Instituto Oceanográfico da Universidade de São Paulo, estão depositadas no Laboratório de Zooplâncton do Departamento de Oceanografia Biológica.

\section{Principais lacunas do conhecimento}

Apesar da abundância dos quetógnatos no zooplâncton de águas brasileiras e de sua importância na trofodinâmica do ecossistema marinho, o grupo continua sendo pouco estudado.

A baixa diversidade apresentada pelo filo no Estado de São Paulo (6,7\% das 209 espécies descritas no mundo) pode ser atribuída, em parte, ao reduzido número de estudos taxonômicos e sinecológicos, sendo estes em sua maioria contribuições isoladas. Isso significa que a sistemática do filo encontra-se ainda em estágios iniciais. Mesmo em outras regiões do mundo, onde o conhecimento taxonômico do filo está bastante adiantado, novas espécies vêm sendo descritas.

Outro problema no tocante ao conhecimento do filo é a baixa representatividade de habitats amostrados. Nesse sentido, estudos detalhados da distribuição batimétrica desses organismos é necessário que sejam feitos porque, com certeza, revelaria a ocorrência de maior número de espécies no Estado de São Paulo. A análise das publicações existentes evidencia que a maioria das espécies estudadas foi coletada no litoral norte, em profundidades menores que $200 \mathrm{~m}$, existindo poucos registros disponíveis para as regiões de Santos e Cananéia, no litoral sul. Assim, é essencial que sejam intensificados os estudos nesses locais e em regiões oceânicas de grandes profundidades, o que pode aumentar o número de espécies do filo no estado. Há ainda os problemas de metodologia de coleta, principalmente a relacionada ao equipamento e embarcações utilizados, que também devem ser levados em consideração porque diminuem a chance de captura desses organismos.

A falta de uma coleção de referência das espécies de Chaetognatha do Estado de São Paulo é um problema sério porque dificulta muito os trabalhos de identificação e, conseqüentemente, o conhecimento do estado atual da biodiversidade do grupo.

Com relação à biodiversidade da fauna de Chaetognatha bentônicos, até o momento não se tem conhecimento de sua ocorrência no estado. No entanto, recentemente, Ávila et al. (2006) 
registram a presença de dois espécimes de Paraspadella (Spadellidae), na divisa dos Estados da Bahia e Espírito Santo, em 2004.

Por outro lado, o número reduzido de trabalhos que tratam da flutuação natural das populações de espécies conhecidas e do monitoramento faunístico, não permite detectar espécies em extinção, invasoras ou introduzidas e endemismo. Este último, contudo, é pouco provável que seja evidenciado uma vez que as espécies identificadas em águas brasileiras possuem ampla distribuição nos oceanos do mundo.

\section{Perspectivas de pesquisa em filo Chaetognatha para os próximos 10 anos}

O número de espécies que ocorrem em águas brasileiras está longe de ser totalmente conhecido devido, principalmente, ao reduzido número de pesquisadores dedicados ao estudo do grupo. Portanto, é necessário incentivar a formação de especialistas, mediante a concessão de bolsas de estudo e de políticas que favoreçam sua permanência no Estado de São Paulo, para que possam dar continuidade aos estudos dos componentes deste importante grupo.

Esforços devem ser feitos para a criação e manutenção de coleções didáticas e de referência, o que facilitaria o trabalho dos pesquisadores dedicados ao estudo taxonômico da fauna de Chaetognatha. O fácil acesso às espécies que ocorrem em águas brasileiras e no estado de São Paulo, em particular, aumentaria o interesse de alunos de iniciação científica e de pós-graduação pelo grupo. Além disso, é importante estimular o desenvolvimento de estudos taxonômicos com material já coletado e que ainda não foi analisado.

Ênfase deve ser dada ao estudo dos quetógnatos bentônicos, a fim de poder inventariar as espécies que ocorrem em São Paulo, uma vez que ainda são totalmente desconhecidas. O mesmo deve ser feito com as que habitam maiores profundidades, estuários, mangues e marismas, porque é muito provável que outras espécies venham a ser encontradas. Para atingir essas metas é fundamental que projetos multidisciplinares, ou mesmo individuais, que tenham como objetivo o estudo de invertebrados marinhos e que apresentem planejamento minucioso recebam apoio financeiro. Atenção especial deve ser dada à infra-estrutura necessária às coletas desses organismos, as quais exigem utilização de equipamento específico (redes, dragas, CTD, etc.), embarcações de médio e grande porte, bem como a participação de técnicos especializados para realização dos trabalhos de campo e laboratório. Apoio financeiro também deve ser dado para a modernização de laboratórios, equipamentos para análise das amostras e para publicação dos resultados em revistas internacionais e nacionais.

\section{Referências Bibliográficas}

ALMEIDA-PRADO, M.S. 1961a. Chaetognatha encontrados em águas brasileiras. Bolm Inst. Oceanogr., S Paulo 11(2):31-56.

ALMEIDA-PRADO, M.S. 1961b. Distribuição dos Chaetognatha no Atlântico Sul Ocidental. Bolm Inst. oceanogr., S Paulo 11(4):15-49.

ALMEIDA-PRADO, M.S. 1963. Sobre o plâncton da Enseada do Mar Virado e os métodos de coleta. Bolm Inst. oceanogr., S Paulo 12(3):49-68.

ALMEIDA-PRADO, M.S. 1968. Distribution and annual occurrence of Chaetognatha of Cananéia and Santos coast (São Paulo, Brazil). Bolm Inst. Oceanogr., S Paulo 17(1):33-55.

ALMEIDA-PRADO, M.S.P. 1960. A new species of Sagitta from the southern Brazilian coast. Bolm Inst. oceanogr., S Paulo 32(2):275-280.

ALVARIÑO, A. 1963. Quetognatos epiplanctónicos del Mar de Cortés. Rev Soc. Mex. Hist. Nat 24:97-203.

ALVARIÑO, A. 1965. Chaetognaths. Oceanogr. Mar. Biol. a. Rev. 3:115-194

ALVARIÑO, A. 1969. Los quetognatos del Atlántico: distribución y notas esenciales de sistemática. Trab. Inst. esp. Oceanogr., (37):1-290.
ALVARIÑO, A. 1980. El plancton del Atlántico suroeste, dinamica y ecologia. Bolm Inst. Oceanogr., S Paulo 29(2):15-26.

ALVARIÑO, A. 1985. Predation in the plankton realm, mainly with reference to fish larvae. Invest. Mar. CICIMAR 2:1-122.

ARAÚJO, H.M.P. \& RIBEIRO, V.A. 2005. Distribuição das espécies de Chaetognatha na plataforma continental de Sergipe e Alagoas. Braz. J. Aquat. Sci. Technol. 9(1):19-23.

ÁVILA, L.R.M., ARRUDA, M.R. \& BONECKER, S.L.C. 2006. Chaetognatha. In Atlas de zooplâncton da região central da zona econômica exclusiva brasileira (S.L.C. Bonecker, ed.). Museu Nacional, Rio de Janeiro, p.165-184.

BALDASSERONI, V. 1915. Reccolte planctoniche fatte dalla R. "Nave Liguria" nel viaggio di circonnavigazione del 1903-1905. Chaetognati. Pubbl. Ist. Studi. Sup. Prat. Firenze 2:85-118.

BIERI, R. 1959. The distribution of the planktonic Chaetognatha in the Pacific and their relationship to the water masses. Limnol. Oceanogr. 4:1-28. http://dx.doi.org/10.4319/1o.1959.4.1.0001

BIERI, R. 1991. Systematics of the Chaetognatha. In The biology of chaetognaths. (Q. Bone, H. Kapp \& A.C. Pierrot-Bults, eds.). Oxford University Press, Oxford.

BOLTOVSKOY, D. 1981a. Chaetognatha. In Atlas del zooplancton del Atlántico Sudoccidental y métodos de trabajo con zooplancton marino. (D. Boltovskoy, ed.). Inst. Nacional Invest. Desarrollo Pesquero, INIDEP, Mar del Plata, p.759-791.

BOLTOVSKOY, D. 1981b. Pteropoda and Chaetognatha identified in a plankton collection from the South Western Atlantic Ocean. Physis 39(97):1-54.

BONE, Q., KAPP, H. \& PIERROT-BULTS, A.C. 1991. The biology of chaetognaths. Oxford University Press, Oxford.

BRUSCA, R.C. \& BRUSCA, G.J. 2007. Invertebrados. 2. ed. Editora Guanabara Koogan, Rio de Janeiro.

BURFIELD, S.T. 1930. Chaetognatha. In British Antarctic ("Terra Nova") Expedition, 1910, Natural history report: zoology. Londres, Trustees of the British Museum 7:203-228.

CASANOVA, J.P. 1999. Chaetognatha. In South Atlantic Zooplankton. (D. Boltovskoy, ed.). Backhuys Publishers, Leiden, The Netherlands, v.2, p.1353-1374.

CASANOVA, J.P., HERNÁNDEZ, F. \& JIMÉNEZ, S. 2006. Spadella lainezi n. sp., the first cave chaetognath from the Eastern Atlantic Ocean. Vieraea 34:17-24.

CASANOVA, J-P. 1985. Les chaetognathes de la Mer Rouge: remarques morphologiques et biogéographiques, description de Sagitta erithraea sp. n. Rapport et Procés-verbaux des Réunions de la Commission internationale pour l'exploration scientifique de la Mer Méditerranée, 29:269-274.

CASANOVA, J-P. 1986. Archeterokrohnia rubra n. gen, n. sp., nouveau chaetognathe abyssal de l'Atlantique nord-africain: description et position systématique, hypothèse phylogénétique. Bull. Mus. Nat. Hist. Natur. Paris, sér. 4, 8:185-194

CASANOVA, J-P. 1987. Deux chaetognathes benthiques nouveaux du genre Spadella de parages de Gibraltar. Remarques phylogénétiques. Bull. Mus. Nat. Hist. Natur., Paris, sér. 4. 9:375-390.

CHENEY, J. 1985a. Spatial temporal abundance patterns of oceanic chaetognaths in the western north Atlantic I. Hydrographic and seasonal abundance patterns. Deep-Sea Res. 32(9):1041-1059. http://dx.doi. org/10.1016/0198-0149(85)90061-5

CHENEY, J. 1985b. Spatial and temporal abundance patterns of oceanic chaetognaths in the western north Atlantic II. Vertical distribution and migrations. Deep-Sea Res. 32(9):1061-1075. http://dx.doi. org/10.1016/0198-0149(85)90062-7

COELHO, M.J. 1993. Zooplâncton do Atlântico Sudoeste (2759'S a 3959'S;

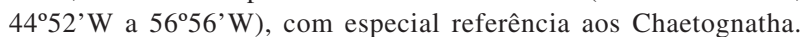
Dissertação de mestrado, Universidade de São Paulo, São Paulo. 
COSTA, P.F. 1970. Nota preliminar sobre a ocorrência de Sagitta friderici e S. enflata (Chaetognatha) na baía de Guanabara. Públ. Inst. Pesq. Mar. 47:1-15.

COSTA, P.F. 1971. Chaetognatha encontrados entre Victória e Ilha da Trindade. Publ. Inst. Pesq. Mar. 51:1-31.

DAPONTE, M.C., GIL DE PERTIERRA, A.A., PALMIER, M.A. \& STROWSKI DE NUÑEZ, M. 2008. Monthly occurrence of parasites of the chaetognath Sagitta friderici off Mar del Plata, Argentina. J. Plankt. Res. 30(5):567-576. http://dx.doi.org/10.1016/j.icesjms.2004.03.006

DEL PRADO-ROSAS, M.C.G., ÁLVAREZ-CADENA, J.N., SEGURAPUERTAS, L. \& LAMOTHE-ARGUMEDO, R. 2005. Hemiurid Metacercariae (Trematoda) in Chaetognaths from the Mexican Caribbean Sea. Comp. Parasitol. 72(2):230-233.

DILLING, L. \& ALLDREDGE, A.L. 1993. Can chaetognatha fecal pellets contribute significantly to carbon flux? Mar. Ecol. Prog. Ser. 92:51-58. http://dx.doi.org/10.3354/meps092051

DUCRET, F. 1968. Chaetognathes des campagnes de "L'Ombango" dans les eaux équatoriales et tropicales africaines. Cah. O.R.S.T.O.M., sér. Océanogr. 6(1):96-141.

DURÓ, A. \& GILI, J.M. 2000. Vertical distribution and abundance of juvenile chaetognaths in the Weddll Sea (Antarctica). Polar Biol. 24:66-69.

FAGUETTI, E.G. 1968. Quetognatos de la expedición "Marchile I" con observaciones acerca del posible valor de algunas especies como indicadoras de las massas de agua frente a Chile. Revista de Biologia Marina, Valparaiso, 13: 85-171.

FEIGENBAUM, D.L. \& MARIS, R.C. 1984. Feeding in the Chaetognatha. Oceanogr. Mar. Biol. Ann. Rev. 22:343-392.

GHIRARDELLI, E. 1968. Some aspects of the biology of chaetognaths. In Advances in Mar. biol. (F.S. Russel \& M.Yonge, eds) v.6, p.271-375.

GUSMÃO, L.M.O. 1986. Chaetognatha planctônicas de províncias nerítica e oceânica do Nordeste do Brasil (04 graus 00' 00" - 08 graus 00' 00") latitude sul. Dissertação de mestrado, Universidade Federal de Pernambuco, Recife.

HOSOE, K. 1956. Chaetognatha from the Isles of the Fernando de Noronha. Contrção Avulsa Univ. S Paulo 3:1-9.

HOSSFELD, B. 1996. Distribution and biomass of arrow worm (Chaetognatha) in Golfo de Nicoya and Golfo Dulce, Costa Rica. Rvta Biol. Trop. 44(3):157-172.

HYMAN, L.H. 1959. The invertebrates. Smaller Coelomate groups. McGrawHill, New York, v.5, p.1-71.

KASATKINA, A.P. \& SELIVANOVA, E.N. 2003. New species of arrowworms (Chaetognatha) belonging to genera Oligoradiata, Leptosagitta, and Mesosagitta from Kievka Bay, Sea of Japan. Russ. J. Mar. Biol. 29(3):144149. http://dx.doi.org/10.1023/A:1024612514599

KUHL, W. 1938. Chaetognatha. In Bronn's Klassen und Ordnungen des Tierreich, Band 4, Vermes, Abteilung 4, Buch2, Teil1, p.1-226.

LIANG, T.H. \& VEGA-PÉREZ, L.A. 1994. Studies on chaetognaths of Ubatuba region. I. Distribution and abundance. Bolm Inst. Oceanogr., S Paulo 42(1-2):73-84.

LIANG, T.H. \& VEGA-PÉREZ, L.A. 1995. Studies on chaetognaths of Ubatuba region, Brazil. II. Feeding habits. Bolm Inst. Oceanogr., S Paulo 43(1):27-40.

LIANG, T.H. \& VEGA-PÉREZ, L.A. 2001. Diversity, abundance, and biomass of epiplanktonic chaetognath off South Atlanctic Western sector, from Cabo Frio (230 S, 420 W) to São Pedro and São Paulo Rocks (01o N, 29o W). Oceanides 16(1):34-48.

LIANG, T.H. \& VEGA-PÉREZ, L.A. 2002. Distribution, abundance and biomass of chaetognaths off São Sebastião region, Brazil, in February 1994. Rev. Bras. Oceanogr. 50(único):1-12.

LIANG, T.H. 1993. Ocorrência e distribuição do filo Chaetognatha na região de Ubatuba, litoral norte do Estado de São Paulo. Dissertação de mestrado, Universidade de São Paulo, São Paulo.
LIANG, T.H. 1998. Estudo dos Chaetognatha epiplanctônicos da região de São Sebastião $\left(23^{\circ} 30^{\prime} \mathrm{S}-24^{\circ} 25^{\prime} \mathrm{S}, 45^{\circ} 55^{\prime} \mathrm{W}-44^{\circ} 55^{\prime} \mathrm{W}\right)$ e da região compreendida entre os Penedos de São Pedro e São Paulo $\left(01^{\circ} \mathrm{N}, 29^{\circ} \mathrm{W}\right)$ e o Cabo Frio $\left(23^{\circ} \mathrm{S}, 41^{\circ} 55^{\prime} \mathrm{W}\right)$. Tese de doutorado, Universidade de São Paulo, São Paulo.

LIANG, T.H., ARA, K., MIRANDA, L.B., BÉRGAMO, A.L. \& BERNARDES, M. 2003. On the variability of the chaetognath Sagitta friderici Ritter-Záhony at Cananéia Lagoon Estuarine System, São Paulo, Brazil. Hydrobiologia 510:91-102. http://dx.doi.org/10.1023/ B:HYDR.0000008635.53746.dd

LOUREIRO F.L., STERZA, J.M. \& NEVES, K. DE O. 2005. Seasonal chaetognath abundance and distribution in a tropical estuary (Southeastern, Brazil). Braz. J. Oceanogr. 53(1-2):47-53.

MARAZZO, A. \& NOGUEIRA, C.S.R. 1996. Composition, spatial and temporal variations of Chaetognatha in Guanabara Bay Brazil. J. Plankt. Res. 18(12):2367-2376. http://dx.doi.org/10.1093/plankt/18.12.2367

MARAZZO, A., MACHADO, C.F. \& NOGUEIRA, C.S.R. 1997. Notes on feeding Chaetognatha in Guanabara Bay Brazil. J. Plankt. Res. 19(7):819828. http://dx.doi.org/10.1093/plankt/19.7.819

McLELLAND, J.A. \& HEARD, R.W. 1991. Notes on some chaetognaths from Pine Cay, Turks and Caicos Islands (British West Indies). Gulf Res. Repts. 8(3):227-235.

McLELLAND, J.A. 1984. Observations on the chaetognaths distributions in the northeastern Gulf of Mexico during the summer of 1974. Northeast Gulf Sci. 7:49-59.

NAGASAWA, S. \& MARUMO, R. 1977. Seasonal variation in composition and number of epipelagic chaetognaths in Sagami Bay, Japan. Le Mer 15:185-195.

NAIR, V.R. \& SANKARANKUTTY, C. 1988. Chaetognaths of the Potengi Estuary (Natal, Northeast, Brazil). Atlantica, Rio Grande, 10(1):5-20.

OCEAN BIOGEOGRAPHIC INFORMATION SYSTEM - OBIS. Www. iobis.org/ (último acesso em 25/05/2010).

ØRESLAND, V. \& BRAY, R.A. 2005. Parasites and headless Chaetognaths in the Indian Ocean. Mar. Biol. 147:725-734.

ØRESLAND, V. 1990. Feeding and predation impact of the chaetognatha Eukrohnia hamata in Gerlach Strait, Antarctic Peninsula. Mar. Ecol. -Progr. Ser. 63:201-209. http://dx.doi.org/10.3354/meps063201

OWEN, R.W. 1981. Fronts and eddies in the sea: mechanisms, interactions and biological effects. In Analyses of marine ecosystems. (A.R. Longhurst, ed.). Academic Press, p.197-233.

OWRE, H.B. 1960. Plankton of Florida Current. The Chaetognath. Bull. Mar. Sci., Gulf Carib, 10(3):255-322.

PEARRE JUNIOR, S. 1976. A seasonal study of the diets of three sympatric chaetognaths. Investigación Pesquera, Barcelona, 40:1-16.

PEARRE JUNIOR, S. 1980. Feeding by Chaetognatha: the relation of prey size to predator size in several species. Mar. Ecol.-prog. Ser.,3:125-134.

PIERROT-BULTS, A.-C \& NAIR, V.R. 1991. Distribution patterns in Chaetognatha. In The biology of chaetognaths (Q. Bone, H, Kapp \& A.C. Pierrot-Bults, eds), Oxford University Press, Oxford, p.86-116.

PIERROT-BULTS, A.C. 1996. Chaetognatha. In Introducción al estudio del zooplancton marino. (R. Gasca \& E. Suárez, eds). México. ECOSUR/ CONACYT, p.529-596.

QUOY, J.R.C. \& GAIMARD, P. 1827. Observations zoologiques faites à bord de l'Astrolabe en Mai 1826, dans le detroit de Gibraltar. Ann. Sci. Natur. Zool., 10:5-239."

REEVE, M.R. \& WALTER, M.A. 1972. Observations and experiments on methods of fertilization in the chaetognath Sagitta hispida. Biological Bulletin, 143: 207-214.

REEVE, M.R. 1970. Complete cycle of development of a pelagic chaetognath in culture. Nature. London, 227: 381-381.

RESGALLA, J.R.C. \& MONTÚ, M. 1995. Quetógnatos de la plataforma continental del sur de Brasil. Inv. Mar. CICIMAR 10(1-2):23-41. 
RUSSEL, F.S. 1939. Hydrographical and biological conditions in North Sea as indicated by plankton organisms. J. Cons. perm. int Explor. Mer, 14:171-192.

SOUSA, L.C.A. \& LOPES, M.J.S. 1998. Estudo dos Chaetognatha da região norte, área compreendida entre a foz do Rio Pará/PA à baía de São Marcos/ MA - Revizee n.1.

TERAZAKI, M. \& MARUMO, R. 1982. Feeding habits of meso- and bathypelagic Chaetognatha, Sagitta zetesios Fowler. Oceanologica Acta, 5: 461-464.

TERAZAKI, M. 1998. Life history, distribution, seasonal variability and feeding of the pelagic chaetognath Sagitta elegans in the Subarctic Pacific: a review. Plankton Biol. Ecol. 45:1-17.

THIEL, M.E. 1938. Die Chaetognathen Bevölkerung des Südatlantischen Ozean. Wissenschaftliche Ergebnisse der deutschen atlantischen Expedition auf dem Vermessungs- und Forschungsschiff "Meteor" 1925-27, 13:1-110.

TOVAR, E. \& SUÁREZ-MORALES, E. 2007. New records and a new species of Spadella (Chaetognatha: Spadellidae) from the western Caribbean Sea. Proc. Biol. Soc. Wash. 120(2):175-183. http://dx.doi.org/10.2988/0006324X(2007)120[175:NRAANS]2.0.CO;2
VANUCCI, M. \& HOSSOE, K. 1952. Resultados científicos do cruzeiro "Baependi" e do "Vega" à Ilha da Trindade. Chaetognatha. Bolm Inst. Oceanogr., S Paulo, 3(1-2):1-31.

VEGA-PÉREZ, L.A. \& LIANG, T.H. 1992. Feeding of Sagitta friderici RitterZáhony, off Ubatuba region (São Paulo, Brazil). Bolm Inst. oceanogr., S Paulo, 40(1-2):93-100.

VEGA-PÉREZ, L.A. \& LIANG, T.H. 1999. Filo Chaetognatha. In Estado do conhecimento da biodiversidade dos invertebrados marinhos do Estado de São Paulo. (A.C. Yoli, ed.). Fundação de Amparo à Pesquisa do Estado de São Paulo, São Paulo, p.17-22.

VEGA-PÉREZ, L.A. \& LIANG, T.H. 2004. Hábitos alimentares dos Chaetognatha da região de São Sebastião, Estado de São Paulo, Brasil. In Simpósio Brasileiro de Oceanografia. CD Rom.

VEGA-PÉREZ, L.A. \& SCHINKE, K.P. 2008. Zooplâncton. In Influência do complexo estuarino da Baixada santista sobre o ecossistema da Plataforma adjacente - ECOSAN. (A.M.S. Pires-Vanin, coordenadora). Rel. no 4 (julho 2007 - junho 2008), cap 5. p.270-306.

WORLD REGISTER OF MARINE SPECIES - WORMS: www. marinespecies.org/ (último acesso em 25/05/2010).

Recebido em 23/07/2010

Versão reformulada recebida em 10/10/2010

Publicado em 15/12/2010 


\section{Apêndice}

Apêndice 1. Referências complementares.

Appendix 1. Complementary references.

ALVAREZ-CADENA, J.N. 1993. Feeding of the Chaetognath Sagitta elegans Verrill. Est. Coast. Sh. Sci. 36(2):195-206. http://dx.doi.org/10.1006/ecss.1993.1013 ALVARIÑO, A. 1962. Two new Pacific Chaetognatha: their distribution and relationship to allied species. Bull. Scripps Instn. Oceanogr. Tech, Ser. 8:1-50. ALVARIÑO, A. 1967. The Chaetognatha of the NAGA Expedition (1956-1961) in the South China Sea and the Gulf of Thailand. Part 1 - Systematics. NAGA Rep. 4(2):1-88.

ALVARIÑO, A. 1978. Spadella gaetanoi, a new benthic Chaetognath from Hawaii. Proc. Biol. Soc. Wash. 91(3):650-657.

ALVARIÑO, A. 1983. The depth distribution, relative abundance and structure of the population of Chaetognatha Alvariño, 1962 in the California Current off California and Baja California. An. Inst. Cienc. Mar Limnol. Univ. nac. autón. México 10:47-84.

BAIER, C.T. \& PURCELL, J.E. 1997. Trophic interactions of chaetognaths, larval fish, and zooplankton in the South Atlantic Bight. Mar. Ecol.-Progr. Ser. 146:43-53. http://dx.doi.org/10.3354/meps146043

BAIER, C.T. \& TERAZAKI, M. 2005. Interannual variability in a predator-prey interaction: climate, chaetognaths and copepods in the southeastern Bering Sea. J. Plankt. Res. 27(11):1113-1125.

BAINBRIDGE, V. 1963. Continuous plankton records: contribution towards a plankton atlas of the Chaetognatha. Bull. Mar. Ecol. 6(2):40-51.

BIGELOW, H.B. 1926. Plankton of the offshore water of the Gulf of Maine. Bull. Bur. Fish. Wash., Doc. 968(40):1-509.

BOLTOVSKOY, D. 1975. Some biometrical, ecological, morphological and distributional aspects of Chaetognatha. Hydrobiologia 46(4):515-534. http:// dx.doi.org/10.1007/BF00028289

BRANDINI, F.P., LOPES, R.M., GUTSEIT, K.S., SPACH, H.L. \& SASSI, R. 1997. Planctologia na plataforma continental do Brasil - Diagnose e revisão bibliográfica. Avaliação do Potencial Sustentável de Recursos Vivos na Zona Econômica Exclusiva- REVIZEE.

CAMIÑAS, J.A. 1985. Quetognatos del Mar del Alborán (Resultados de la Campaña “Málaga 775”). Boln Inst. Esp. Oceanogr. 2(1).

CADENA, J.N.A., MENDIVIL, A.R.A., LÓPEZ, U.O. \& SABIDO, A.U. 2008. Composición, abundancia y distribución de las especies de quetognatos del litoral norte del Caribe de México. Hidrobiológica 18(1):37-48.

CASANOVA, J.P. 1991. The first record of a benthic polar chaetognath: a new Spadella from the Antarctic. J. Nat. Hist. 25:1355-1362. http://dx.doi. org/10.1080/00222939100770841

CASANOVA, J.P. 1993. Spadella japonica, a new coastal benthic chaetognath from Japan. Proceedings of the biological remarks. Cahiers de Biologie Marine, 45:373-379.

CASANOVA, J.P. \& NAIR, V.R. 1999. A new species of the genus Sagitta (Phylum Chaetognatha) from the Agatti lagoon (Laccadive Archipelago, Indian Ocean) with comments on endemism. Ind. J. Mar. Sci. 29:169-172.

CASANOVA, J.P. \& NAIR, V.R. 2002. A new species of Sagitta (Chaetognatha) from a Laccadive lagoon (Indian Ocean) having fan-shaped anterior teeth: phylogenetical implications. J. Nat. History 36:149-156. http://dx.doi.org/10.1080/00222930010009345

CASTELLÕES, P.V. 2000. Distribuição vertical do filo Chaetognatha em um ponto fixo da Baía de Guanabara (RJ-Brasil). Dissertação de mestrado, Universidade Federal do Rio de Janeiro, Rio de Janeiro.

CRELIER, A.M. \& DAPONTE, M.C. 2004. Chaetognatha of the Brazil-Malvinas (Falkland) confluence: distribution and associations. Iheringia, Sér. Zool. 94(4):403-412.

DAPONTE, M.C., CAPITANIO, F.L., NAHADEBIAN, D.E., VIÑAS, M.D. \& NEGRI. R.M. 2004. Sagitta friderici Ritter-Záhony (Chaetognatha) from South Atlantic waters: abundance, population structure, and life cycle. ICES J. Mar. Sci. 61:680-686. http://dx.doi.org/10.1016/j.icesjms.2004.03.006

DAWES, B. 1958. Sagitta as a host of larval trematodes, including a new and unique type of cercaria. Nature 182(4640):960-961. PMid:13590199. http:// dx.doi.org/10.1038/182960a0

DRITS, A.V. 1981. Some patterns of feeding of Sagitta enflata. Oceanology 21:624-628.

DURÓ, A., SABATÉS, A \& GILI, J.M. 1999. Mesoscale spatial distribution of chaetognath along hidrographic gradients in the South Scotia Sea (Antarctica). Polar Biol. 22:195-206. http://dx.doi.org/10.1007/s003000050410

DUVERT, M., PEREZ, Y. \& CASANOVA, J.P. 2000. Wound healing and survival of beheaded chaetognaths. J. Mar. Biol. Ass. U.K. 80:891-898. http://dx.doi. org/10.1017/S0025315400002873

FOWLER, G.H. 1963. The Chaetognatha of the "Siboga" Expedition with a discussion of the synonymy and distribution of the group. Siboga-Exped. 21.

FURNESTIN, M.L. \& CODACCIONI, J.C. 1968. Chaetognathes du nor-ouest de L'Océan Indien, (Golfe d'Aden, mer d'Arabie, Golfe d'Oman, Golfe Persique). Cah. O.R.S.T.O.M., sér. Océanogr. 6(1):143-171.

GIANESELLA-GALVÃO, S.M.F., AIDAR, E., VEGA-PÉREZ, L.A \& SALDANHA-CORRÊA, F.M.P. 1997. Distribuição do plâncton na região costeira de São Sebastião (SP). In Oceanografia da Plataforma Interna de São Sebastião - OPISS. Relatório de Cruzeiros e Resultados Preliminares. Relat. Tec. Inst. oceanogr. (41):1-13.

GIESECKE, R. \& GONZÁLEZ, H.E. 2008. Reproduction and feeding of Sagitta enflata in the Humboldt Current system off Chile. ICES J. Mar. Sci. 65:361370. http://dx.doi.org/10.1093/icesjms/fsn030

GRANT, G.C. 1991. Chaetognatha from the central and southern Middle Atlantic Bight. Species composition, temperature-salinity relationships and interespecific association. Fish Bull. Natl. Fish Serv. US 89:33-40.

GUSMÃO, L.M.O. 2000. Comunidades zooplanctônicas nas províncias neríticas e oceânicas de Pernambuco - Brasil. Tese de doutorado, Universidade Federal de Pernambuco, Recife.

HAGEN, W. 1985. On the distribution and population structure of Antarctic Chaetognatha. Meeresforsch - Rep. Mar. Res. Sonderdruckaus 30:280-291.

JOHNSON, T.B. \& TERAZAKI, M. 2003. Species composition and depth distribution of chaetognaths in a Kuroshio warm-core ring and Oyashio water. J. Plankt. Res. 25(10):1279-1289. http://dx.doi.org/10.1093/plankt/fbg085

KASATKINA, A.P. 2001. On changes in the arrowworm (Chaetognatha) fauna in the western Bering Sea and northwestern Pacific. Inv. Zool. 27(1):14-20. http://dx.doi.org/10.1023/A:1024612514599 
KEHAYIAS, G. 2003. Quantitative aspects of feeding of chaetognaths in the eastern Mediterranean pelagic waters. J. Mar. Biol. Ass. U.K. 83:559-569. http:// dx.doi.org/10.1017/S0025315403007483h

KIMMERER, W.J. 1984. Selective predation and its impact on prey of Sagitta enflata (Chaetognatha). Mar. Ecol. Progr. Ser.15:55-62. http://dx.doi.org/10.3354/ meps015055

LOPES, R.M., MONTÚ, M.A., GORRI, C.H., MUXAGATA, E., MIYASHITA, L. \& OLIVEIRA, L.P. 2006. O zooplâncton marinho na região entre Cabo de São Tomé (RJ) e o Chui (RS). In O ambiente oceanográfico da Plataforma Continental e do talude na região sudeste-sul do Brasil (C.L.B. RossiWongtschowski \& L.S.P. Madureira, eds.). p.265-358.

McLELLAND, J.A. 1980. Notes on the northern Gulf of Mexico occurrence of Sagitta friderici Ritter-Záhony (Chaetognatha). Gulf Res. Repts. 6:343-348. McLELLAND, J.A. 1989. An illustrated key to the Chaetognatha of the northern Gulf of Mexico with notes on their distribution. Gulf Res. Repts 8(2):145-172. MILLE-PAGAZA, S. \& CARRILLO-LAGUNA, J. 2001. The chaetognatha of the southwestern Gulf of Mexico during April-May, 1986. Gulf and Caribb. Res. 13:59-65.

MILLE-PAGAZA, S., REYES-MARTÍNEZ, R. \& SÁNCHEZ-SALAZAR, M.E. 1997. Distribution and abundance of chaetognatha on the Yucatan shelf during May, 1986. Gulf. Res. Rept. 9(4):263-275

MORENO, I. 1973. Clave de la identificación de los quetognatos de los mares de la Península Ibérica. Bol. Inst. Esp. Oceanogr. Madrid. 159:1-29.

MOSTAJO, E. 1976a. Clave para la determinación de los quetognatos del Atlântico sudoccidental. Neotropica (Argentina) 68:81-86.

MOSTAJO, E. 1976b. Quetognatos de "Pesqueria V". Su utilización como indicadores hidrológicos. Iheringia, Zool. 49:27-38.

MURAKAMI, A. 1959. Marine biological study on the planktonic chaetognaths in the Seto Inland Sea. Bull. Naikai reg. Fish Res Lab. 12:1-186.

NAGASAWA, S. 1985. Copulation in the neritic chaetognath Sagitta crassa. J. Plankt. Res. 7(6):927-935. http://dx.doi.org/10.1093/plankt/7.6.927

NAIR, V.R. 1978. Bathymetric distribution of Chaetognaths in the Indian Ocean. Indian J. Mar. Sci. 7:276-282.

NOBLEZADA, M.M.P. \& CAMPOS, W.L. 2008. Spatial distribution of chaetognaths off the northern Bicol Shelf, Philippines (Pacific coast). ICES J. Mar. Sci. 65:484-494. http://dx.doi.org/10.1093/icesjms/fsn027

ØRESLAND, V. 1986. Parasites of the chaetognath Sagitta setosa in western English Channel. Mar. Biol. 92:87-91. http://dx.doi.org/10.1007/BF00392750

ØRESLAND, V. 1987. Feeding of chaetognaths, Sagitta elegans and S. setosa at different seasons in Gullmarsfjorden, Sweden. Mar. Ecol. -Progr. Ser. 39:6979. http://dx.doi.org/10.3354/meps039069

PAKHOMOV, E.A., PERISSINOTTO, R. \& FRONEMAN, P.W. 1999. Predation impact of carnivorous macrozooplankton and micronekton in the Atlantic sector of the Southern Ocean. J. Mar. Systems 19:47-64.

PALMA, S. \& SILVA, N. 2004. Distribution of siphonophores, chaetognaths, euphausiids and oceanographic conditions in the fjords and channels of southern Chile. Deep-Sea Res. II(51):513-535. http://dx.doi.org/10.1016/j.dsr2.2004.05.001

PEARRE JUNIOR, S. 1982. Feeding by Chaetognatha: aspects of inter- and intra-specific predation. Mar. Ecol. -Progr. Ser. 7:33-45.

PEARRE JUNIOR, S. 1991. Growth and reproduction. In The biology of chaetognaths (Q. Bone, H. Kapp \& A.C. Pierrot-Bults, ed.). Oxford University Press, Oxford, p.61-75.

PIERCE, E.L. 1953. The Chaetognatha oure. The continental shelf of north Carolina with attention to their relation to the hydrography of the area. J. Mar. Res. 12(1):75-92.

PIERROT-BULTS, A.C. \& CHIDGEY, K.C. 1988. Chaetognatha. In Synopsis of the British Fauna (New Series) (D.M. Kermack \& R.S.K. Barner, eds). Linn. Soc. London, Leiden, n.39., 66p.

PIERROT-BULTS, A.C. \& NAIR, V.R. 1991. Distribution patterns in Chaetognatha. In The biology of Chaetognatha (Q. Bone, H. Kapp \& A.C. Pierrot-Bults, eds). Oxford University Press, Oxford, p.86-116.

PIERROT-BULTS, A.C. 2007. Chaetognatha of the World. http://nlbif.eti.uva.nl/bis/chaetognatha.php.

RITTER-ZÁHONI, R.V. 1909. Chaetognatha. Denk. Akad. Wissensch. Wien 84:1-18. (Instituto de Biociências - USP)

RITTER-ZÁHONI, R.V. 1909. Die Chaetognathen der Expedition S.M. Sch. "Pola" in das Rote Meer. Denk. Akad. Wissensch. Wien, 84:43-54.

RITTER-ZÁHONI, R.V. 1911. Die Chaetognathen der Plankton - Expedition. Erg. Plankton Exp. 2(2):1-33. (IOUSP / IBUSP).

RUPPERT, E.E., FOX, R.S. \& BARNES, R.D. 2005. Zoologia dos invertebrados. Uma abordagem funcional - evolutiva. Editora Roca, Rio de Janeiro, p.991-998.

SALLES, A.C.R. 2009. Ecologia trófica do extrato juvenil de peixes carangídeos do infralitoral raso da enseada de Caraguatatuba, São Paulo. Dissertação de mestrado, Universidade de São Paulo, São Paulo.

SARDIÑA, P. \& CAZORLA, A.L. 2005. Trophic ecology of the whitemouth croaker, Micropogonias furnieri (Pisces: Sciaenidae), in south-western Atlantic waters. J. Mar. Biol. Ass. U.K. 85:405-413.

SOLOVEV, K.A. \& KOSOBOKOVA, K.N. 2003. Feeding of Chaetognaths Parasagitta elegans Verrill (Chaetognatha) in the White Sea. Oceanology 43(4):524-531

SUND, P.N. 1961. Two new species of Chaetognatha from the waters off Peru. Pacif. Sci. 15:105-111.

TERBIYIK, T., CEVIK, C., TOKLU-ALICLI, B. \& SAIHAN, E. 2007. First record of Ferosagitta galerita (Dallot, 1971) [Chaetognata] in the Mediterranean Sea. J. Plankt. Res. 29(8):721-726.

THUESEN, E.V. 2005. Chaetognatha. http://academic.evergreen.edu/t/thuesene/chaetognaths/chaetognaths.htm.

TOKIOKA, T. 1962. The outline of the investigations made on chaetognaths of the Indian Ocean. Inform. Bull. Plankt. Japan 8:5-11.

TOKIOKA, T. 1965. The taxonomical outline of chaetognaths. Publications of the Seto Marine Biological Laboratory 12:335-357.

ULLOA, R., PALMA, S. \& SILVA, N. 2004. Relationship between spatial distribution of chaetognaths and oceanographic conditions off Concepción Bay, Chile. Deep-Sea Res II(51):537-550. http://dx.doi.org/10.1016/j.dsr2.2004.05.002

VEGA, F.R. 1964. Sistematica y consideraciones sobre la distribucion de Chaetognatha en Vera Cruz. Mexico. Tese para obtención del título de Biologo. Universidad Autónoma de México.

VEGA-PÉREZ, L.A. 1993. Estudo do zooplâncton da região de Ubatuba, Estado de São Paulo. Publção esp. Inst. oceanogr., S Paulo, (10):65-84.

VEGA-PÉREZ, L.A. 2008. Zooplâncton. In Oceanografia de um ecossistema subtropical. Plataforma de São Sebastião, SP. (A.M.S. Pires-Vanin, ed.). São Paulo, EDUSP, p.253-272. 
Vega-Pérez, L.A. \& Schinke, K.P.

VEGA-PÉREZ, L.A. \& SCHINKE, K.P. 2006. Avaliação do zooplâncton do Canal de Bertioga, Estado de São Paulo, no verão. In Simpósio Brasileiro de Oceanografia. CD Rom.

VILLENAS, F. \& PALMA, S. 2006. Sagitta chilensis nueva especie de quetognato en fiordos australes chilenos (Chaetognatha, Aphragmophora, Sagittidae). Invest. Mar., Valparaíso 34(1):101-108.

VILLENAS, F., PALMA, S. \& SOTO, D. 2009. Chaetognath spatial distribution and how egg-carrying affects the vertical distribution of Sagitta tasmanica off southern Chile. Sci. Mar. 73(1):29-38. 